TRANSACTIONS OF THE

AMERICAN MATHEMATICAL SOCIETY

Volume 173, November 1972

\title{
ANALYTICALLY INVARIANT AND BI-INVARIANT SUBSPACES
}

\author{
BY
}

\section{DOMINGO ANTONIO HERRERO( $\left.{ }^{1}\right)$ AND NORBERTO SALINAS}

\begin{abstract}
The purpose of this paper is to call attention to some interesting weakly closed algebras related to a bounded linear operator $T$ acting on a Banach space $\boldsymbol{X}$ and their associated lattices of invariant subspaces, namely, the algebras generated by the polynomials and by the rational functions in $T$, and the commutant and the double-commutant of $T$. The relationship between those algebras and their lattices, as well as the ones corresponding to the operators induced by $T$ on an invariant subspace (restriction), or on the quotient space $X / \mathcal{W}$ (where $M$ is an invariant subspace of a given type) are analyzed. Several results relative to the decomposition of invariant subspaces and the topological structure of the lattices (under the "gap-between-subspaces" metric topology) are also considered.
\end{abstract}

1. Introduction. Let $\mathcal{X}$ be a complex Banach space, and let $\mathscr{L}(\mathcal{X})$ denote the algebra of all (bounded linear) operators on $X$.

For each $T \in \mathscr{L}(\mathcal{X})$, there are several weakly closed algebras (with identity) canonically associated with $T$; namely,

$\mathscr{Q}_{T}$, the algebra generated by the polynomials in $T$;

$\mathfrak{U}_{T}^{a}$, the algebra generated by the analytic functions in $T$;

(1.1) $\mathfrak{U}_{T}^{\prime}$, the commutant of $T$ (i.e., $\mathfrak{X}_{T}^{\prime}=\{S \in \mathscr{Q}(\mathfrak{X}): S T=T S\}$ ); and

$\mathscr{U}_{T}^{\prime \prime}$, the double commutant (or bi-commutant) of $T$

$$
\text { (i.e., } \mathfrak{U}_{T}^{\prime \prime}=\left\{R \in \mathfrak{L}(\mathfrak{X}): S R=R S \text {, for every } S \in \mathfrak{U}_{T}^{\prime}\right\} \text { ). }
$$

Likewise, there are lattices of (closed) subspaces of $\mathcal{X}$ naturally related to $T$; namely

$$
g_{T}, \quad g_{T}^{a}, \quad g_{T}^{\prime \prime} \text { and } \mathcal{I}_{T}^{\prime}
$$

corresponding to the invariant subspace lattice of the algebra $\mathfrak{U}_{T}$, $\mathfrak{U}_{T}^{a}$, $\mathcal{U U}_{T}^{\prime \prime}$, and

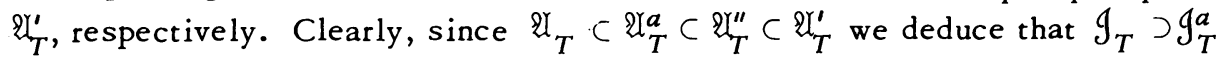

Received by the editors April 12, 1971.

AMS (MOS) subject classifications (1970). Primary 47A15, 46L20; Secondary 46L10, 47A25, 47B20.

Key words and phrases. Lattices of invariant, analytically invariant, bi-invariant, hyperinvariant subspaces, complementary invariant subspaces, decomposition, polynomial。 ly generated algebra, analytically generated algebra, commutant, double commutant, reflexive algebra, relatively reflexive algebra, splitting algebra, splitting lattice, gap-metric topology for invariant subspaces, restriction, quotient, normal operators, subnormal operators, minimal normal extension.

(1)The first author was supported by National Science Foundation grant GU-3171. 
$\supset \mathcal{I}_{T}^{\prime \prime} \supset \mathcal{I}_{T}^{\prime}$. Observe that, from a classical result, if $\mathcal{X}$ is finite dimensional, $\mathscr{U}_{T}=$

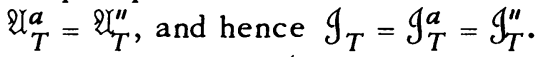

The lattices $\mathscr{I}_{T}$ (invariant subspaces of $T$ ) and $\mathscr{I}_{T}^{\prime}$ (hyperinvariant subspaces of $T$ ) have been studied in detail by several authors ([6], [18]) while $\mathcal{I}_{T}^{a}$ and $\mathcal{I}_{T}^{\prime \prime}$ were considered only in very special cases ([3], [4]).

The main purpose of this note is to call attention to the lattices $\mathcal{I}_{T}^{a}$ and $\mathcal{G}_{T}^{\prime \prime}$ and to point out some facts about them which may be useful in the study of the structure of $\mathscr{I}_{T}$ and $\mathcal{I}_{T}^{\prime}$.

Definition 1.1. The members of $g_{T}^{a}$ will be called analytically invariant subspaces of $T$, and those of $\mathcal{G}_{T}^{\prime \prime}$ bi-invariant subspaces of $T$.

In $\$ 2$ we shall find a simple characterization of the analytically invariant subspaces of an operator $T$ and we shall give a useful sufficient condition for a subspace to be bi-invariant under $T$.

Douglas and Pearcy (in [6]) asked whether $\mathscr{I}_{T}=\mathscr{I}_{T}^{\prime}$ implies $\mathfrak{U}_{T}=\mathfrak{U}_{T}^{\prime}$. The same type of problems arise by considering the different lattices of (1.2). In fact, in $\$ 2$ we give examples showing that all kinds of situations are possible with regard to equality or inequality of the algebras (1.1). However, in all these examples, the equality of a pair of lattices in (1.2) corresponds to equality in the corresponding algebras in (1.1). Also, in $\$ 3$, we discuss some other cases in which the same phenomenon occurs.

In $\$ 4$ we generalize to Banach spaces the results of Douglas and Pearcy [6] and, in $\$ 5$, we prove a decomposition theorem for $\mathcal{I}_{T}^{a}, \mathcal{I}_{T}^{\prime \prime}, \mathcal{I}_{T}^{\prime}$ analogous to that obtained by Douglas and Pearcy for $\mathscr{I}_{T}$. We also generalize a result of Crimmins and Rosenthal [5] concerning a decomposition of $g_{T}$.

Finally, in $\$ 6$, we discuss some facts concerning restrictions of operators and related topics. The case of subnormal operators on Hilbert space is specially considered.

2. Analytically invariant subspaces and examples. In what follows the term subspace will refer to a closed linear manifold and by an algebra we shall mean a weakly closed algebra with identity.

If $\mathscr{U}$ and $\mathfrak{B}$ are two subalgebras of $\mathscr{L}(X)$ such that $\mathscr{U} \subset \mathfrak{B}$, and $\sigma(T ; \mathscr{X})$ $(\sigma(T ; \mathbb{B}))$ denotes the spectrum of the operator $T$ with respect to $\mathscr{U}(\mathbb{B})$, then it is not difficult to show that

$$
\partial \sigma(T ; \mathfrak{X}) \subset \sigma(T ; \mathfrak{B}) \subset \sigma(T ; \mathfrak{U})
$$

(where $\partial X$ denotes the boundary of the set $X$ ). Moreover, it is easily seen that the algebra $\mathfrak{U}_{T}^{a}$ can be characterized as follows:

Lemma 2.1. Let $T \in \mathfrak{L}(\mathscr{X})$. Then $\mathfrak{U}_{T}^{a}$ is the minimal subalgebra $\mathfrak{U}$ of $\mathscr{L}(\mathscr{X})$ satisfying $\sigma(T ; \mathfrak{U})=\sigma(T)$. 
Proof. If $f(z)$ is a function defined and analytic in a neighborhood of $\sigma(T)$, then it follows from the well-known theorem of Runge (see [12]) that $f(T)$ (defined by means of the Dunford-Riesz functional calculus [11, Chapter XI]) can be approximated in the norm by finite linear combinations of operators of the form $\left(T-\lambda_{k}\right)^{-1}, \lambda_{k} \in \rho(T)$ (the resolvent set of $T$ ).

It follows that $\mathfrak{U}_{T}^{a}$ is equal to the weakly closed algebra generated by the operators $T$ and $(T-\lambda)^{-1}, \lambda \in \rho(T)$. Using this characterization, the result follows immediately. Q.E.D.

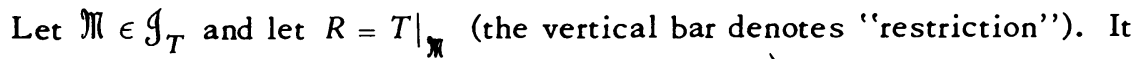
is well known [5] that $\partial \sigma(R) \subset \sigma(T)$. Therefore, $\sigma(R) \backslash \sigma(T)$ consists of some bounded components of the complement $\rho(T)$ of $\sigma(T)$. In the case when $\mathbb{N}$ is analytically invariant, the following characterization can be given:

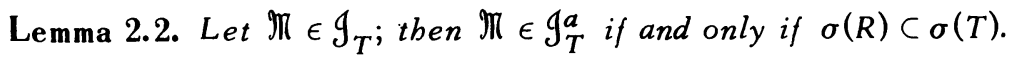

Proof. Assume that $\mathbb{R} \in \mathcal{G}_{T}^{a}$ and let $\lambda \in \rho(T)$; then $(T-\lambda) \mathbb{M} \subset \mathbb{M},(T-\lambda)^{-1} \mathbb{M}$ C M and therefore

$$
\mathbb{M}=(T-\lambda) \mathbb{M}=(T-\lambda)^{-1} \mathbb{M} .
$$

It is not hard to conclude that $(R-\lambda)$ is invertible and, moreover,

$$
(R-\lambda)^{-1}=\left.(T-\lambda)^{-1}\right|_{\pi} \text {. }
$$

Hence, $\lambda \in \rho(R)$; i.e., $\sigma(R) \subset \sigma(T)$.

On the other hand, if $\mathbb{M} \in \mathcal{G}_{T}$ and $\sigma(R) \subset \sigma(T)$, then $(R-\lambda)$ is invertible in $\mathbb{N}$ and its inverse coincides with $\left.(T-\lambda)^{-1}\right|_{M}$, for all $\lambda \in \rho(T)$.

Therefore, $(T-\lambda) \mathbb{M}=\mathbb{M}=(T-\lambda)^{-1} \mathbb{M}$, for all $\lambda \in \rho(T)$. The result follows from Lemma 2.1. Q.E.D.

D. Sarason [14] has already proved that if $\lambda$ belongs to the unbounded component of $\rho(T)$, then $I_{(T-\lambda)-1}=g_{T}$ and, if $\lambda_{1}, \lambda_{2}$ belong to the same components of $\rho(T)$, then $\mathscr{I}_{\left(T-\lambda_{1}\right)-1}=\mathscr{I}_{\left(T-\lambda_{2}\right)-1}$. Hence

$$
g_{T}^{a}=g_{T} \cap\left[\bigcap_{n} g_{\left(T-\lambda_{n}\right)-1}\right] \text {, }
$$

where the (possible empty) set $\left\{\lambda_{n}\right\}$ contains exactly one (arbitrary) point of each bounded component of $\rho(T)$. All these results can be obtained as applications of the above-mentioned the orem of Runge. Another consequence of Runge's theorem is the fact that, if $\rho(T)$ is connected, then $\mathfrak{U}_{T}^{a}=\mathfrak{U}_{T}$. However, $\mathfrak{U}_{T}^{a}$ could be equal to $\mathfrak{U}_{T}$ even if $\rho(T)$ is disconnected.

Example 1. Let $S$ be the unilateral shift (multiplication by $e^{i x}$ ) in the Hardy class $H^{2}$ of analytic functions in the unit disc; let $q$ be an inner function 
and let $T$ be the operator in $\left(q H^{2}\right)^{\perp}$ defined by $T f=P S f\left(f \in\left(q H^{2}\right)^{\perp}\right)$, where $P$ denotes the orthogonal projection of $H^{2}$ onto $\left(q H^{2}\right)^{\perp}$. Then (see [13]) $\mathfrak{U}_{T}^{\prime}=\mathfrak{U}_{T}$.

On the other hand (see [6, Lecture VIII]), it is known that $\sigma(T)$ consists of exactly those complex numbers $\lambda \in D=\{z:|z|<1\}$, such that $q(\lambda)=0$ and all those $\lambda \in \partial D$, such that $q(z)$ cannot be continued analytically to $z=\lambda$. Thus, for suitably chosen $q, \rho(T)$ is disconnected, but $\mathfrak{U}_{T}^{a}=\mathfrak{U}_{T}^{\prime}=\mathfrak{U}_{T}$.

The following result provides a way to construct bi-invariant and hyperinvariant subspaces:

Lemma 2.3. Let $T \in \mathfrak{L}(\mathfrak{X}), A \in \mathfrak{U}_{T}^{\prime}$ and $B \in \mathfrak{U}_{T}^{\prime \prime}$, and let $\mathbb{M}$ be an invariant subspace of $T$. Then the following bold:

(i) $\operatorname{kernel}(A) \in \mathcal{I}_{T}^{\prime \prime}, \operatorname{kernel}(B) \in \mathcal{I}_{T}^{\prime}$;

(ii) closure $[A \mathbb{M}] \in \mathcal{I}_{T}$; moreover, if $\mathbb{M} \in \mathcal{I}_{T}^{a}\left(\right.$ or $\left.\mathcal{I}_{T}^{\prime \prime}\right)$, then closure [AM] $\in \mathcal{I}_{T}^{a}$ $\left(\mathcal{G}_{T}^{\prime \prime}\right.$, resp. );

(iii) if $\mathbb{M} \in \mathcal{I}_{T}^{\prime}$, then closure $[B \mathbb{M}] \in \mathfrak{I}_{T}^{\prime}$;

(iv) in particular, if $X$ is a Hilbert space, then every reducing subspace of $T$ is bi-invariant.

Proof. The assertions follow after a straightforward computation. For (iv), observe that $\mathbb{M}$ is a reducing subspace of $T$ if and only if $T P=P T$, where $P$ is the orthogonal projection of $\mathcal{X}$ onto $\mathbb{M}$; i.e., if and only if $P \in \mathfrak{U}_{T}^{\prime}$. Finally, according to (i), $\mathbb{M}=P X \in \mathcal{I}_{T}^{\prime \prime}$. Q.E.D.

We do not know whether this lemma gives all the bi-invariant and hyperinvariant subspaces or not. However, this is true for some particular operators.

Let $T$ be a normal operator acting on a Hilbert space $\mathcal{H}$; by a well-known result about von Neumann algebras (see, e.g., [16]), $\mathfrak{U}_{T}^{\prime \prime}$ is equal to the weakly closed algebra generated by $T$ and $T^{*}$ (the adjoint of $T$ ). Therefore $\mathcal{I}_{T}^{\prime \prime}$ is precisely the set of all subspaces which are invariant under $T$ and $T^{*}$; i.e.,

$$
\begin{aligned}
\mathscr{S}_{T}^{\prime \prime} & =\{\mathbb{M}: \mathbb{M} \text { reduces } T\}=\left\{\mathbb{M}=\operatorname{ker} P: P \in \mathfrak{U}_{T}^{\prime}\right\} \\
& =\left\{\mathbb{M}=\text { closure }[P \mathcal{H}]: P \in \mathfrak{U}_{T}^{\prime}\right\} .
\end{aligned}
$$

On the other hand, the hyperinvariant subspaces of $T$ are the kernels of the spectral projections of $T$ (see [6, p. 330]); that is to say,

$$
\mathscr{I}_{T}^{\prime}=\left\{\mathbb{M}=\operatorname{ker} P: P \in \mathfrak{U}_{T}^{\prime \prime}\right\}=\left\{\mathbb{M}=\operatorname{clos}[P \mathcal{H}]: P \in \mathfrak{U}_{T}^{\prime \prime}\right\}
$$

Moreover, we may only consider the orthogonal projections contained in $\mathfrak{U}_{T}^{\prime}$ (for $\mathfrak{I}_{T}^{\prime \prime}$ ) and in $\mathfrak{U}_{T}^{\prime \prime}$ (for $\mathfrak{I}_{T}^{\prime}$ ).

Similarly, if $\mathcal{X}$ is a finite-dimensional space, then

$$
\mathscr{I}_{T}=\mathscr{I}_{T}^{\prime \prime}=\left\{\mathbb{M}=\operatorname{ker} A: A \in \mathfrak{U}_{T}^{\prime}\right\}=\left\{\mathbb{M}=A \mathfrak{X}: A \in \mathfrak{U}_{T}^{\prime}\right\}
$$


and $\mathcal{I}_{T}^{\prime}$ is the lattice generated by the subspaces

$$
\left\{\mathbb{M}: \mathbb{M}=\operatorname{ker} A \text { or } \mathbb{M}=A \mathfrak{X} \text {, for some } A \in \mathfrak{X}_{T}^{\prime \prime}\right\} \text {. }
$$

In the next three examples, we shall be concerned with normal operators $w$ ith a cyclic vector; these operators can be described, up to unitary equivalence, as follows:

Let $\mu$ be a positive Borel measure on the complex plane $\mathbf{C}$ with compact support $K$; then the operator $T\left(=M_{z}\right)$ defined in the Hilbert space $\mathcal{H}=L^{2}(K, \mu)$ as the multiplication by the function $e_{1}(z)=z$ is bounded and normal. Moreover, if $e_{0}(z)=1$ (a.e. $d \mu$ ), then $\left(T^{*}=M_{\bar{z}}\right)$,

$$
\mathcal{H}=\bigvee\left\{T^{* n} T^{m} e_{0}: n, m=0,1,2, \cdots\right\}=\left\{B e_{0}: B \in \mathfrak{U}_{T}^{\prime \prime}\right\}
$$

(the sign " $\bigvee$ " means "the subspace spanned by") and $\mathfrak{U}_{T}^{\prime}=\mathfrak{U}_{T}^{\prime \prime}$ (see [16]).

Example 2. Let $T$ and $\mathcal{H}$ be as above with $K=\{z:|z|=1\}$ and $\mu=$ linear Lebesgue measure on $K$. Then $T$ is unitary and therefore

$$
\left(T^{-1}=T^{*}\right) \quad \mathfrak{U}_{T}^{a}=\mathscr{U}_{T}^{\prime \prime}=\left\{M_{f}: f \in L^{\infty}(K, \mu)\right\} .
$$

However, $\mathfrak{U}_{T}^{a} \neq \mathfrak{U}_{T}$; in fact, we have

$$
\mathcal{I}_{T}^{a}=\mathcal{I}_{T}^{\prime \prime}=\mathcal{I}_{T}^{\prime}=\left\{L^{2}(E, \mu): E \text { is a measurable subset of } K\right\},
$$

but (see [9])

$$
\mathcal{I}_{T}=\mathcal{G}_{T}^{a} \cup\left\{q H^{2}: q \in L^{\infty}(K, \mu) ;\left|q\left(e^{i x}\right)\right|=1 \text { (a.e.) }\right\} .
$$

Example 3. If $K=\{z:|z| \leq 1\}$ and $d \mu$ is the planar Lebesgue measure, then $\sigma(T)=K$ and therefore $\mathfrak{U}_{T}^{a}=\mathfrak{U}_{T}$. As in the previous case, we have

$$
\mathfrak{U}_{T}^{\prime}=\mathfrak{X}_{T}^{\prime \prime}=\left\{M_{f}: f \in L^{\infty}(K, \mu)\right\} \text {. }
$$

In this case $\mathfrak{Q I}_{T}^{a} \neq \mathfrak{U}_{T}^{\prime \prime}$; in fact,

$$
\mathbb{M}=\bigvee\left\{B\left(e_{0}\right): B \in \mathfrak{U}_{T}\right\} \in \mathcal{I}_{T}=\mathscr{I}_{T}^{a}
$$

is the closure in $L^{2}(K, \mu)$ of the polynomials in $z$. It is not hard to see (use, e.g., $[12$, p. 286]) that, if $f(z) \in \mathbb{M}$, then there exists a function $g(z)$ analytic in the open unit disc, such that $f(z)=g(z)$ (a.e. $d \mu)$. Therefore, $\mathbb{M}^{\prime} \mathcal{J}_{T}^{\prime \prime}\left(M_{\bar{z}} \mathbb{M}\right.$ $\not \subset \mathbb{M})$ and we conclude that $\mathscr{I}_{T}^{a} \neq \mathscr{I}_{T}^{\prime \prime}$; hence $\mathfrak{U}_{T}^{a} \neq \mathfrak{U}_{T}^{\prime \prime}$.

Example 4. If, in the above example, the unit disc is replaced by $K_{0}=$ $\{z: 1 / 2 \leq|z| \leq 1\}$, then $\mathfrak{U}_{T} \neq \mathfrak{U}_{T}^{a} \neq \mathfrak{U}_{T}^{\prime \prime}=\mathfrak{U}_{T}^{\prime}$ and $\mathscr{I}_{T} \neq \mathfrak{I}_{T}^{a} \neq \mathscr{I}_{T}^{\prime \prime}=\mathscr{I}_{T}^{\prime}$ (it is enough to consider the subspaces generated by the function $e_{0}$, as in the previous cases).

Examples 1 to 4 show that all the cases of (1.1) satisfying the condition $\mathfrak{U}_{T}^{\prime \prime}=\mathfrak{U}_{T}^{\prime}$ are actually possible. To see that the other four cases are also 
possible, it suffices to take "two copies" of the same example; e.g., if $T$ and $\mathcal{H}$ are as in Example 2, and $U=T \oplus T$ is the normal operator acting on $\mathcal{X}=\mathcal{H} \oplus \mathcal{H}$ (direct sum with the norm $\left.\left\|\left(f_{1}, f_{2}\right)\right\|_{\mathscr{X}}^{2}=\left\|f_{1}\right\|_{\mathcal{H}}^{2}+\left\|f_{2}\right\|_{\mathcal{H}}^{2}\right)$ defined by $U\left(f_{1}, f_{2}\right)=$ $\left(T f_{1}, T f_{2}\right)$, then

$$
\mathfrak{U}_{U} \neq \mathfrak{U}_{U}^{a}=\mathfrak{U}_{U}^{\prime \prime} \neq \mathfrak{U}_{U}^{\prime} \text { and } \mathscr{I}_{U} \neq \mathscr{I}_{U}^{a}=\mathscr{G}_{U}^{\prime \prime} \neq \mathscr{I}_{U}^{\prime} \text {. }
$$

In fact, $\mathfrak{X}_{U}=\left\{B \oplus B: B \in \mathfrak{X}_{T}\right\}$ and a similar result is true for $\mathfrak{X}_{U}^{a}=\mathfrak{U}_{U}^{\prime \prime}$, but

$$
\mathfrak{U}_{U}^{\prime}=\left\{\left(\begin{array}{ll}
A & B \\
C & D
\end{array}\right): A, B, C, D \in \mathfrak{U}_{T}^{\prime}\right\} \text {. }
$$

Hence, $\mathcal{H} \oplus\{0\} \in \mathcal{I}_{U}^{a}=\mathcal{I}_{U}^{\prime \prime}, \mathcal{H} \oplus\{0\} \notin \mathcal{I}_{U}^{\prime}$.

3. Relatively reflexive algebras. Given a subalgebra $\mathfrak{U}$ of $\mathscr{L}(\mathscr{X})$, we shall represent by $\mathfrak{Q}^{\wedge}$ the algebra of all operators that leave invariant all the invariant subspaces of $\mathfrak{U}$. The algebra $\mathfrak{U}$ is called reflexive [7] if $\mathfrak{U}=\mathfrak{U}^{\wedge}$.

Definition 3.1. Let $\mathfrak{A}$ and $\mathfrak{B}$ be two subalgebras of $\mathscr{L}(\mathscr{X})$. We shall say that $\mathfrak{U}$ is relatively reflexive with respect to $\mathscr{P}$ whenever $\mathfrak{U}^{\wedge} \cap \mathfrak{B}=\mathfrak{U} \cap \mathbb{B}$.

Thus, an algebra is reflexive if and only if it is relatively reflexive with respect to any subalgebra of $\mathscr{L}(\mathscr{X})$. Moreover, if $\mathfrak{U}$ is relatively reflexive with respect to $\mathscr{R}$, then it is also relatively reflexive with respect to any subalgebra contained in $\mathscr{R}$.

Definition 3.2. We shall say that the algebra $\mathscr{U} \subset \mathcal{Q}(\mathscr{X})$ has the property $(\mathscr{P})$ if, given $x_{1}, x_{2}, \ldots, x_{n} \in \mathcal{X}$, there exists $x \in \mathcal{X}$ such that

$$
\left\|T x_{j}\right\| \leq\|T x\|, \text { for all } T \in \mathfrak{U} \text {. }
$$

Remark. If $\mathfrak{U}$ has the property $(\mathcal{P})$, then every subalgebra $\mathfrak{S}$ of $\mathfrak{U}$ has also the property $(\mathscr{P})$. Furthermore, it is easy to see that, if $\mathscr{X}$ is the direct sum of the finite of Banach spaces $\left\{\mathcal{X}_{n}\right\}_{n=1}^{N}$ (under any norm) and $\mathfrak{X}_{n}$ is a subalgebra of $\mathscr{L}\left(\mathscr{X}_{n}\right)$ satisfying the property $(\mathscr{P})$ for each $n=1,2, \ldots, N$, then the subalgebra $\mathfrak{U}$ of $\mathscr{L}(\mathscr{X})$ defined by $\mathfrak{U}=\bigoplus_{n=1}^{N} \mathfrak{U}_{n}$ also satisfies the property $(\mathscr{P})$.

Theorem 3.1. If $\mathfrak{A}$ bas the property $(\mathcal{P})$, then every subalgebra $(\mathfrak{S} \subset \mathfrak{U}$ is relatively reflexive with respect to 2 .

Proof. Let $\mathcal{I}$ be the lattice of invariant subspaces of $\mathbb{S}$ and let $T \in \mathfrak{U}$ be any operator such that $I_{T} \supset \mathcal{G}$. Let $x_{1}, x_{2}, \ldots, x_{n} \in \mathcal{X}$ and let $\epsilon>0$ be given. If $x \in \mathcal{X}$, then $\mathfrak{X}_{x}=\bigvee\{L x: L \in \mathbb{S}\} \in \mathcal{I} \subset \mathscr{I}_{T}$; therefore, $T x \in \mathfrak{X}_{x}$ and there exists an operator $L \in \mathbb{S}$ such that $\|(T-L) x\|<\epsilon$.

If $x$ is chosen satisfying $(3.1)$, it follows that (observe that $(T-L) \in \mathfrak{U}^{\prime}$ )

$$
\left\|(T-L) x_{j}\right\|<\epsilon, \quad \text { for } j=1,2, \cdots, n .
$$


Hence, $T$ belongs to the strong closure of $(S)$ and therefore (since $\sqrt{5}$ is weakly closed) $T \in$ (S. Q.E.D.

Examples 5 (Algebras with the property $(\mathcal{P})$ ).

I. Let $\mathcal{C}(K)$ be the Banach algebra of all continuous functions on a compact set $K$ (with the usual "supremum" norm). Let $\mathscr{M}(K)=\left\{M_{f}: M_{f}\right.$ is the multiplication by $f \in \mathcal{C}(K)\}$; then, if $\mathcal{X}$ is a subspace of $\mathcal{C}(K)$ containing a function $b$ such that $|b(k)| \geq \delta>0$, for all $k \in K$, and $\mathfrak{X}=\left\{M_{f} \in \mathfrak{M}(K): f g \in \mathcal{X}\right.$, for all $\left.g \in \mathfrak{X}\right\}$, then $\mathcal{U}$ has the property $(\mathcal{P})$. (In fact, given $g_{1}, g_{2}, \cdots, g_{n} \in \mathcal{X}$, the function $g=$ $(1 / \delta)\left(\sum_{j=1}^{n}\left\|g_{j}\right\|_{\infty}\right) b \in \mathcal{X}$ satisfies (3.1).)

II. Let $\Phi(t)$ be an increasing positive convex function defined for nonnegative real values of $t$ such that $\Phi(0)=0$ and $\lim \Phi(t) / t \geq 1(t \rightarrow+\infty)$; let $(M, \mu)$ be a measure space and let $\mathcal{X} L^{\Phi}(M, \mu)$ be the corresponding Orlicz space. Let $\mathfrak{U}=\left\{M_{f}: M_{f}\right.$ is the multiplication by $\left.f \in L^{\infty}(M, \mu)\right\}$; then $\mathfrak{U}$ has the property $(\mathcal{P})$ (given $g_{1}, g_{2}, \ldots, g_{n} \in \mathcal{X}, g(k)=\max \left\{\left|g_{j}(k)\right|: j=1,2, \ldots, n\right\}$ (a.e. $d \mu$ ) satisfies (3.1)).

III. Let $\left\{\mathscr{X}_{a}, \mathfrak{X}_{a}\right\}_{a \in \Xi}$ be a family of Banach spaces and algebras of the type described in II and let $\mathcal{X}$ be the topological direct sum of the $\mathcal{X}_{a}$ 's (under some "suitable" norm, e.g., $\left\|\left\{f_{a}\right\}\right\|_{\mathscr{X}}=\sup _{a}\left\|f_{a}\right\|_{\boldsymbol{X}_{\alpha}} ;\left\|\left\{f_{\alpha}\right\}\right\|_{\mathscr{X}}=\left(\Sigma_{\alpha \in \Xi}\left\|f_{\alpha}\right\|_{\mathscr{X}_{a}}^{r}\right)^{1 / r}, 1 \leq r<$ $\infty ;\left\|\left\{f_{a}\right\}\right\|_{\mathscr{X}}=\sup _{a}\left\|f_{\alpha}\right\|_{\mathscr{X}_{a}}$, and $f_{a}=0$ for all but a denumerable subset of indices, etc.). Let $\mathscr{U}$ be the algebra of all bounded linear operators $T$ in $\mathcal{X}$ such that $\mathfrak{X}_{\alpha} \in \mathcal{I}$ (the lattice of $\mathfrak{U}$ ) for all $\alpha \in \Xi$ and $\left.T\right|_{\mathfrak{X}_{\alpha}} \in \mathfrak{U} \mathfrak{\alpha}_{\alpha}$, then $\mathfrak{U}$ has the property $(\mathfrak{P})$.

IV. Let $\dot{H}$ be a Hilbert space and let $\mathfrak{B}$ be an abelian von Neumann algebra on $\mathcal{H}$. Then $\mathfrak{B}$ is unitarily equivalent to a subalgebra of the multiplication algebra $L^{\infty}(X, \mu)$ acting on $L^{2}(X, \mu)$, where $(X, \mu)$ is an appropriate localizable measure space $[17$, Part II, Theorem 1]. Therefore $\mathfrak{B}$ has the property $(\mathcal{P})$.

Lemma 3.2. If $\mathfrak{Q}$ is an algebra of operators of the type described in Example 5. II and III, then $\mathcal{U}$ is a maximal abelian subalgebra of $\mathfrak{L}(\mathfrak{X})$. Moreover, $\mathfrak{A}$ is reflexive.

Proof. Let $P_{\alpha}$ be the projection of $X$ onto $X_{\alpha}$ and let $M_{\chi_{\alpha}}$ be the operator "multiplication by the characteristic function of $E_{\alpha}$ " (where $E_{\alpha}$ is any $\mu_{\alpha}{ }^{-}$ measurable subset of $M_{\alpha}$; clearly, $P_{\alpha} \in \mathfrak{U}$ and $M_{x_{\alpha}} \in \mathscr{U}{ }_{\alpha}$, for all $\alpha \in \Xi$. Let $B$ $\epsilon \mathscr{L}(\mathcal{X})$ and assume that either $B$ commutes with $P_{a}{ }^{M_{1}}{ }_{a} P$ or $B$ leaves invariant each of the subspaces $M_{x_{\alpha}} X_{a}=L^{\Phi} a\left(E_{\alpha^{\prime}} d \mu_{\alpha}\right)$, for all $\alpha \in \Xi$ and for all $\mu_{\alpha^{-}}$ measurable $E_{\alpha} \subset M_{\alpha}$. In both cases, we obtain the same conclusion: If $f_{a}$ is a simple function of $\mathcal{X}_{\alpha}$, then there exists a $\mu_{\alpha}$-measurable function $g_{\alpha}$ such that $B(f)=g_{a} P_{a}(f)$ ( $f$ being the element $\left\{f_{\gamma}\right\}$, where $f_{\gamma}=f_{a}$ if $\gamma=a, f_{\gamma}=0$ for $\gamma \neq$ a). By a density argument, we conclude that $B \in \mathfrak{N}$. Q.E.D. 
Several observations of $A$. Lambert made it possible to state the present version of the following two results, instead of the weaker statements that appeared in the preprint:

Theorem 3.3. Let $\mathfrak{A}$ be a reflexive subalgebra of $\mathscr{L}(\mathscr{X})$ and assume that $\mathfrak{B}^{\wedge}$ $\cap \mathfrak{U}=\mathfrak{B}$ for every algebra $\mathfrak{B} \subset \mathfrak{A}$. Then $\mathfrak{B}$ is also reflexive and, moreover, $\mathfrak{S}^{\wedge}$ $\cap \mathbb{B}=\mathbb{E}$ for every subalgebra $\subseteq$ of $\mathbb{B}$.

Proof. Let $T \in \mathscr{L}(\mathscr{X})$ and assume that $\mathscr{I}_{T}$ contains the lattice $\mathscr{I}_{\mathfrak{B}}$ of invariant subspaces of $B$; since $B \subset \mathscr{U}$, it follows that $\mathscr{I}_{T} \supset \mathscr{I}_{\mathfrak{U}}$, the lattice of invariant subspaces of $\mathscr{U}$. By reflexivity, $T \in \mathfrak{U}$.

On the other hand, $B$ is relatively reflexive with respect to $\mathscr{U}$; since $\mathcal{I}_{T} \supset \mathscr{G}_{\mathfrak{B}}$ and $T \in \mathfrak{U}$, we conclude that $T \in \mathscr{R}$. In other words, $\mathscr{D}$ is a reflexive subalgebra of $\mathcal{L}(\mathcal{X})$.

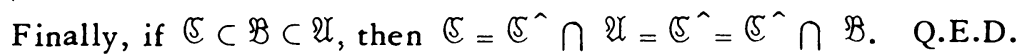

In particular, every subalgebra $\mathscr{O}$ of the type described in Examples 5. II and III satisfies Theorem 3.3. The following result extends a theorem due to D. Sarason [15, Theorem 2]:

Corollary 3.4. (i) If II is as in Examples 5. II or III, then every subalgebra $\mathfrak{B}$ of $\mathscr{U}$ is reflexive.

(ii) In particular, if $T \in \mathfrak{U}$, then equality of two lattices in (1.2) implies equal. ity of the corresponding algebras in (1.1).

(iii) An abelian algebra of normal operators (in a Hilbert space) is always reflexive.

Proof. (i) This is a consequence of Lemma 3.2 and Theorem 3.3.

(ii) For any subalgebras $\mathfrak{X}_{1}, \mathfrak{U}_{2}$ of $\mathfrak{L}(\mathscr{X})$, the inclusion $\mathfrak{U}_{1} \subset \mathfrak{U}_{2}$ implies the inclusions $\mathfrak{U}_{2}^{\prime} \subset \mathfrak{U}_{1}^{\prime}$ and $\mathfrak{U}_{1}^{\prime \prime} \subset \mathfrak{U}_{2}^{\prime \prime}{ }_{2}$ (where $\mathfrak{U}_{j}^{\prime}, \mathfrak{U}^{\prime \prime}{ }_{j}$ denote the commutant and the double commutant of the algebra $\left.\mathfrak{U}_{j}, j=1,2\right)$. Since, by Lemma $3.2, \mathfrak{U}^{\prime}=\mathfrak{U}^{\prime \prime}=\mathfrak{U}$, it follows that

$$
\mathfrak{U}_{T} \subset \mathfrak{U}_{T}^{a} \subset \mathfrak{U}_{T}^{\prime \prime} \subset \mathfrak{U} \subset \mathfrak{U}_{T}^{\prime} .
$$

By (i), $\mathfrak{U}_{T}, \mathfrak{U}_{T}^{a}$ and $\mathfrak{U}_{T}^{\prime \prime}$ are actually reflexive subalgebras of $\mathscr{L}(\mathscr{X})$; thus, if $\mathfrak{U}_{T}^{\prime}=\mathfrak{U}$, we are done. Otherwise, $\mathfrak{U}_{T}^{\prime}$ contains properly $\mathfrak{U}_{\text {and }} \mathfrak{U}_{T}^{\prime \prime}$ is properly

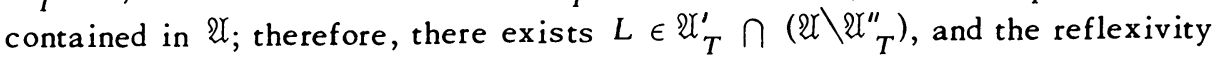
of $\mathscr{Q}$ implies that $\mathscr{I}_{T}^{\prime \prime}$ contains properly the lattice of invariant subspaces of $\mathcal{U}$; since this lattice contains $\mathcal{I}_{T}^{\prime}$, it follows that $\mathcal{I}_{T}^{\prime \prime} \neq \mathcal{I}_{T}^{\prime}$.

(iii) An abelian algebra $B$ of normal operators is always contained in a maximal abelian von Neumann algebra $\mathfrak{B}$; since $\mathfrak{B}$ (see Examples 5 . II and IV) is of the type described in Example 5. II, the result follows from (i). Q.E.D. 
4. A topology for invariant subspaces. Let $\mathcal{H}$ be a Hilbert space and let $T \epsilon$ $\mathcal{L}(\mathcal{H})$; in [6], R. G. Douglas and C. Pearcy studied the structure of $g_{T}$ under the topological structure induced by the metric

$$
\theta(\Re, \Re)=\left\|P_{\Re}-P_{\pi}\right\|
$$

where $\mathbb{N}, \pi$ are subspaces of $\mathcal{H}$ and $P_{\Re}, P_{\pi}$ denote the orthogonal projections of $\mathcal{H}$ onto $\mathbb{N}, \pi$, respectively. Most of their results can be extended to a more general situation; if $\mathcal{X}$ is an arbitrary Banach space, then it is possible to introduce a metric in the set of all subspaces of $X$ which, in a case when $X$ is a Hilbert space, induces the same topology as $\theta$.

Let $\pi, \pi$ be subspaces of $X$; following [10] we define

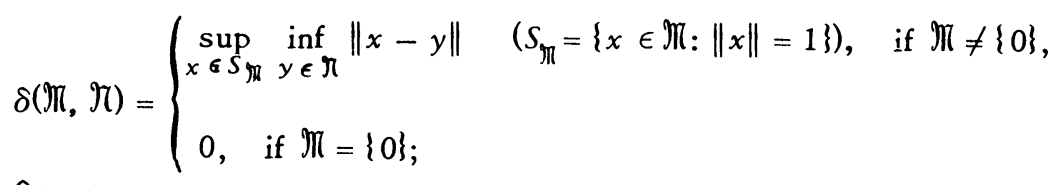

$\hat{\delta}(\Re, \Re)=\max [\delta(\Re, \Re), \delta(\Re, \Re)]$;

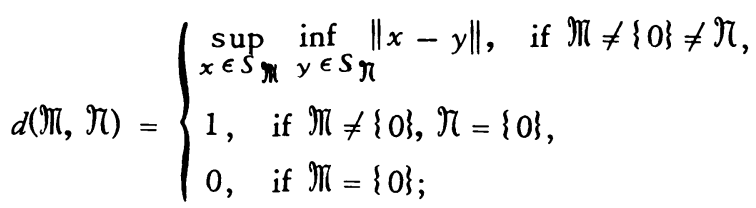

$\hat{d}(\Re, \Re)=\max [d(\pi, \Re), d(\Re, \pi)]$.

As it is well known [10], $\hat{d}$ is a metric in the set of all subspaces of $\mathcal{X}$. Moreover,

$$
\begin{aligned}
& \delta(\Re, \Re) \leq d(\Re, \Re) \leq 2 \delta(\Re, \Re), \quad \text { and } \\
& \hat{\delta}(\Re, \Re) \leq \hat{d}(\Re, \Re) \leq 2 \hat{\delta}(\Re, \Re),
\end{aligned}
$$

for all pairs of subspaces $\Re, \pi$. If $X$ is a Hilbert space, then $\hat{\delta}(\pi, \Re)=\theta(\pi, \Re)$. However, when arbitrary Banach spaces are considered $\hat{\delta}$ is not a metric.

The following results can be obtained from a suitable modification of the proofs given in [6] and [10]; therefore, we shall only sketch the proofs.

Lemma 4.1. Let $\mathfrak{X}$ be a subalgebra of $\mathfrak{L}(\mathscr{X})$ and let $\mathscr{I}$ be its lattice of in. variant subspaces. Then $(\mathfrak{G}, \hat{d})$ is a complete metric space.

Lemma 4.2 (cf. [6, Lemma 1.1]). For $i=1,2$, let $\mathbb{M}_{i}$ be a subspace of $\mathfrak{X}$ and let $C_{i} \in \mathfrak{L}(\mathcal{X})$ satisfy $\left\|C_{i} x\right\| \geq \epsilon_{i}\|x\|\left(\epsilon_{i}>0\right)$ for all $x \in \mathbb{M}_{i}$. If $\boldsymbol{\pi}_{i}=$ $\mathcal{C} \mathbb{M}_{i}, i=1,2$, then $\pi_{i}$ is closed and

$$
\dot{\hat{\delta}}\left(\Re_{1}, \pi_{2}\right) \leq\left(\left\|C_{1}\right\| / \epsilon_{1}+\left\|C_{2}\right\| / \epsilon_{2}\right) \hat{\delta}\left(\Re_{1}, \pi_{2}\right)+\left(1 / \epsilon_{1}+1 / \epsilon_{2}\right)\left\|C_{1}-C_{2}\right\| .
$$




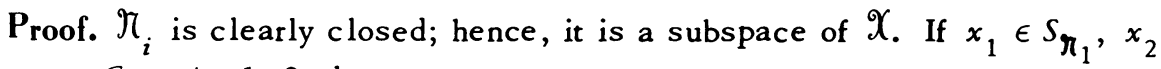
$\epsilon \Re_{2}, x_{i}=C_{i} y_{i}, i=1,2$, then

$$
\begin{aligned}
\inf \left\{\left\|x_{1}-x_{2}\right\|: x_{2} \in \Re_{2}\right\}=\inf \left\{\left\|C_{1} y_{1}-C_{2} y_{2}\right\|: C_{2} y_{2} \in \Re_{2}\right\} \\
\leq \inf \left\{\left\|C_{1}\left(y_{1}-y_{2}\right)\right\|+\left\|\left(C_{1}-C_{2}\right) y_{2}\right\|: y_{2} \in \cdot \mathbb{M}_{2}\right\} .
\end{aligned}
$$

Observe that

$$
\inf \left\{\left\|x_{1}-x_{2}\right\|: x_{2} \in \Re_{2}\right\}=\inf \left\{\left\|x_{1}-x_{2}\right\|: x_{2} \in \Re_{2},\left\|x_{2}\right\| \leq 1\right\} .
$$

Thus, we get

$$
\begin{aligned}
\inf \left\{\left\|x_{1}-x_{2}\right\|: x_{2} \in \pi_{2}\right\} & \leq\left\|C_{1}\right\| \inf \left\{\left\|y_{1}-y_{2}\right\|: y_{2} \in \pi_{2}\right\}+\left(1 / \epsilon_{2}\right)\left\|C_{1}-C_{2}\right\| \\
& \leq\left(\left\|C_{1}\right\| / \epsilon_{1}\right) \delta\left(\pi_{1}, \pi_{2}\right)+\left\|C_{1}-C_{2}\right\| / \epsilon_{2} .
\end{aligned}
$$

Hence,

$$
\delta\left(\pi_{1}, \pi_{2}\right) \leq\left(\left\|C_{1}\right\| / \epsilon_{1}\right) \delta\left(\pi_{1}, \pi_{2}\right)+\left\|C_{1}-C_{2}\right\| / \epsilon_{2} .
$$

From this inequality and the one obtained interchanging the roles of $\pi_{1}$ and $\pi_{2}$, the result follows. Q.E.D.

A point $A$ of a metric space $R$ is called inaccessible if the only cont inuous map $\gamma$ from the closed unit interval $[0,1]$ into $R$ such that $\gamma(0)=A$ is the constant map $\gamma(t)=A, 0 \leq t \leq 1$. Using Lemma 4.2 and the proof of [6, Theorem 1] we obtain

Theorem 4.3. Let $\mathbb{M}$ be an inaccessible point of $\mathcal{G}$. Then $\mathbb{M} \in \mathcal{I}^{\prime}$, the lattice of invariant subspaces of the commutant algebra $\mathfrak{U}^{\prime}$ of $\mathfrak{U}$.

Corollary 4.4 (cf. [6, Corollaries 1.2 and 1.4]). If $\pi \in \mathcal{G}$ is isolated or it is

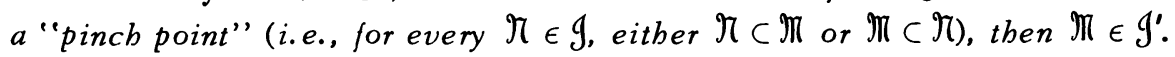

Proof. The result is clear for isolated points. Now, if $\pi \subset \pi$ (or $\pi \subset \pi$ ) and $\Re \neq \pi$, then $\hat{\delta}(\pi, \pi)=1$; hence, every "pinch point" of $I$ is isolated. Q.E.D.

Theorem 4.5 (cf. [6, Theorem 2]). Let $\mathscr{X}$ and $\mathbb{B}$ be two similar subalgebras of $\mathscr{L}(\mathcal{X})$ (i.e., there exists an invertible operator $S \in \mathscr{L}(X)$ such that $T \rightarrow S^{-1} T S$ is a bijective map from $\mathfrak{U}$ onto $\mathfrak{B})$, then the lattices of invariant subspaces of $\mathfrak{U}$ and $\mathfrak{B}$ are bomeomorphic topological spaces.

5. A decomposition of invariant subspaces. Let $\mathscr{U}$ be a subalgebra of $\mathscr{L}(\mathscr{X})$ and let $\mathfrak{U}^{\prime}, \mathfrak{U}^{\prime \prime}$ be its commutant and its double commutant, respectively; then $\mathscr{U} \subset \mathfrak{U}^{\prime \prime}$ ( $\mathfrak{U}^{\prime \prime} \subset \mathfrak{U}^{\prime}$ if and only if $\mathfrak{U}^{\prime \prime}$ is commutative, if and only if $\mathfrak{U}$ is commutative, if and only if $\mathfrak{U}\left(\mathfrak{U}^{\prime}\right)$. Let $\mathscr{J}, \mathfrak{I}^{\prime}, \mathscr{I}^{\prime \prime}$ be the corresponding lattices of invariant subspaces. We shall need two simple lemmas whose proofs are left to the interested reader. 
Lemma 5.1 (generalization of Lemma 2.3). If $A \in \mathcal{U}^{\prime}, B \in \mathfrak{U}^{\prime \prime}, \mathbb{M} \in \mathcal{Y}$ and $\Re \in$ $g^{\prime}$, then

(i) $\operatorname{ker}(A) \in \mathcal{G}^{\prime \prime}, \operatorname{ker}(B) \in \mathcal{I}^{\prime}$;

(ii) $\mathrm{cl}[A \Re] \in \mathcal{I}, \mathrm{cl}[B \mathcal{N}] \in \mathcal{I}^{\prime}$;

(iii) if $\mathbb{M} \in \mathcal{G}^{\prime \prime}$, then $\mathrm{cl}[A M] \in \mathcal{G}^{\prime \prime}$.

Lemma 5.2. Let $P_{1} \in \mathfrak{Q}(\mathscr{X})$ be an idempotent and let $P_{2}=I-P_{1}$; set $\gamma=$ $\max \left(\left\|P_{1}\right\|,\left\|P_{2}\right\|\right)$. Then, for every $\pi, \pi \in I_{P_{1}}\left(=g_{P_{2}}\right)$ we have

$$
(1 / \gamma) \max _{j=1,2} \hat{\delta}\left(P_{j} \mathbb{M}, P_{j} \Re\right) \leq \hat{\delta}(\mathbb{N}, \Re) \leq 2 \gamma \max _{j=1,2} \hat{\delta}\left(P_{j} \mathbb{N}, P_{j}, \mathcal{N}\right) .
$$

Definition 5.1. Let $X_{1}$ and $X_{2}$ be two subspaces of $X_{\text {such that }} X_{=} X_{1} \oplus$ $X_{2}$ (i.e., there exist idempotents $P_{j} \in \mathfrak{L}(\mathscr{X}), j=1,2$, such that $P_{1}+P_{2}=I$ and $X_{j}=P_{j} X_{;} P_{j}$ is called the projection of $X_{\text {onto }} X_{j}$ along $\left.X_{3-j}, j=1,2\right)$. We shall say that a subalgebra $\mathcal{O}$ of $\mathscr{L}(\mathscr{X})$ splits with respect to (w.r.t.) $\mathscr{X}_{1}, \mathscr{X}_{2}$ if there exist subalgebras $\mathfrak{X}_{j}$ of $\mathfrak{L}\left(\mathscr{X}_{j}\right)(j=1,2)$ such that $\mathfrak{U}=\mathfrak{U}_{1} \oplus \mathfrak{X}_{2}$. Also, we shall say that the lattice $\mathfrak{L}$ splits w.r.t. $X_{1}, X_{2}$ if, for every $\mathbb{R} \in \mathfrak{Q}$, we have $\pi=\left(\pi \cap X_{1}\right) \oplus\left(\pi \cap X_{2}\right)$.

Theorem 5.3. Let $X_{1}$ and $X_{2}$ be two subspaces of $X_{\text {such that }} X_{=} X_{1} \oplus X_{2}$; then

(i) A subalgebra $\mathfrak{X}$ of $\mathscr{Q}(\mathscr{X})$ splits w.r.t. $X_{1}, X_{2}$ if and only if $P_{j} \in \mathfrak{X} \cap$ $\mathfrak{U}^{\prime}, j=1,2$;

(ii) a lattice $\mathscr{R}$ splits w.r.t. $X_{1}, X_{2}$ if and only if $\mathscr{R} \subset \mathfrak{I}_{P_{1}}$;

(iii) if $\mathscr{A}$ splits w.r.t. $\mathfrak{X}_{1}, \mathscr{X}_{2}$, so does its lattice $\mathscr{Y}$;

(iv) if $I$ splits w.r.t. $X_{1}, X_{2}$ and $X_{1}, X_{2} \in \mathcal{Y}$, then $X_{1}, X_{2} \in \mathcal{I}^{\prime} ; X_{1}$ and $\mathcal{X}_{2}$ are isolated points of $\mathcal{G}$ and $\mathscr{G}$ is bomeomorphic to the topological product $g_{1} \times g_{2}$, where

$$
g_{j}=\left\{\Re \in G: M \subset X_{j}\right\}
$$

is a lattice of subspaces of $X_{j}, j=1,2$;

(v) if $X_{1}, X_{2} \in I \cap \mathcal{I}^{\prime}$, then $\mathfrak{X}^{\prime}, \mathfrak{X}^{\prime \prime}, \mathcal{I}^{\prime}, \mathcal{I}^{\prime \prime}$ splits w.r.t. $X_{1}, X_{2}$; and $X_{j}$ is an isolated point of $\mathcal{G}^{\prime}$ and $\mathcal{G}^{\prime \prime}, j=1,2$.

Proof. (i) Assume that $\mathscr{X}$ splits w.r.t. $X_{1}, X_{2}$. It is clear that $X_{1}, X_{2} \in \mathscr{I}$ and $I=I_{1} \oplus I_{2}$, where $I_{j} \in \mathscr{U}{ }_{j}, j=1,2$; using these facts it follows that $P_{1}=I_{1}$ $\oplus 0, P_{2}=0 \oplus I_{2} \in \mathfrak{U}$.

Let $A \in \mathfrak{U}$ and $x \in \mathfrak{X}$; then $x=x_{1}+x_{2}$, with $x_{j} \in \mathfrak{X}_{j}$ and $P_{1} A x=$ $P_{1} A\left(P_{1}+P_{2}\right) x=P_{1} A x_{1}+P_{1} A x_{2}=P_{1} A x_{1}=A P_{1} x\left(A x_{2} \in X_{2}\right.$ and therefore $\left.P_{1} A x_{2}=0\right)$. Hence $P_{1}, P_{2} \in \mathfrak{U}^{\prime}$.

Now suppose that $P_{j} \in \mathfrak{U} \cap \mathfrak{U}^{\prime}, j=1,2$. Then $\mathfrak{X}_{j} \in \mathcal{I}$. 
Let $\mathfrak{X}_{j}=\left.\mathfrak{X}\right|_{\mathfrak{X}_{j}}$; then the map $\left.\left.A \rightarrow A\right|_{\mathfrak{X}_{1}} \oplus A\right|_{\mathfrak{X}_{2}}$ is an isomorphism from $\mathfrak{X}$ onto $\mathfrak{U}_{1} \oplus \mathfrak{X}_{2}$, and $\mathscr{X}$ splits w.r.t. $X_{1}, X_{2}$.

(ii) Assume that $\mathbb{R}$ splits w.r.t. $X_{1}, X_{2}$ and let $\mathbb{R} \in \mathcal{R}$. Since $\mathbb{M}=\left(\mathbb{R} \cap X_{1}\right)$ $\oplus\left(\mathbb{N} \cap X_{2}\right)$, there exist idempotents $P^{\prime}{ }_{j} \in \mathscr{L}(\mathbb{N}), j=1,2$, such that $P^{\prime}{ }_{1}+P^{\prime}{ }_{2}=$

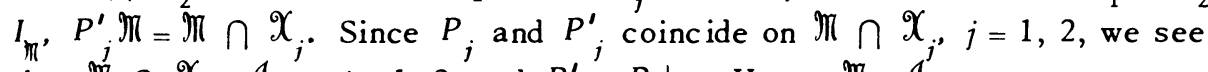
that $\mathbb{M}^{j} \cap X_{j} \in \mathscr{I}_{P_{1}}, j=1,2$, and $P_{j}^{\prime}{ }_{j}^{j}=\left.P_{j}\right|_{\mathbb{M}}$. Hence $\mathbb{M} \in \mathfrak{I}_{P_{1}}$.

The reverse implication is clear.

(iii) We observed in the proof of (i) that $X_{1}, X_{2} \in \mathcal{I}$ and $P_{1}, P_{2} \in \mathscr{U} \cap \mathscr{U}^{\prime}$. Let $\mathbb{M} \in \mathcal{I}$ and let $\mathbb{M}_{j}=\mathbb{M} \cap \mathcal{X}_{j}$; it is clear that $\mathbb{M}_{j} \in \mathcal{I}, j=1,2$. By (ii), $\mathbb{M}, \mathbb{M}_{j}$ $\epsilon \mathscr{I}_{P_{j}}$ and therefore $P_{j} \mathbb{M} \subset \mathbb{M}$; hence $P_{j} \mathbb{M} \subset \mathbb{M}_{j}, j=1,2$. Thus we have

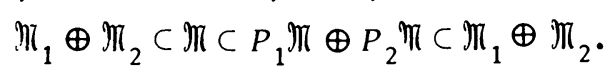

We conclude that $\mathbb{M}_{j}=P{ }_{j} \mathbb{M}_{\text {and }} \mathbb{M}_{=} \mathbb{M}_{1} \oplus \mathbb{M}_{2}$.

(iv) If $\mathcal{I}$ splits w.r.t. $X_{1}^{j}, X_{2}$ and $X_{1}, X_{2} \in \mathcal{I}$, then every $\mathbb{M} \in \mathcal{I}$ can be written as $\mathbb{M}_{1} \mathbb{M}_{1} \oplus \mathbb{M}_{2}$, where $\mathbb{M}_{j}=\mathbb{M}_{\cap} \mathscr{X}_{j} \in \mathcal{I}, j=1$, 2. Therefore, $\mathfrak{I}=\mathscr{I}_{1} \times$ $g_{2}$, where $g_{j}$ is given by $(5.1)$; this equality clearly works both as sets and in the sense of lattices; i.e., $\mathcal{I}$ is lattice-isomorphic to the direct product (as defined in [1]) of the lattices $g_{1}$ and $g_{2}$.

Let $\mathbb{M}_{=} \mathbb{M}_{1} \oplus \mathbb{M}_{2} \in \mathcal{I}$; then

(a) if $\mathbb{M} \subset \mathscr{X}_{1}, \mathbb{M}_{1} \neq \mathscr{X}_{1}$, then $\hat{\delta}\left(\mathbb{M}, x_{1}\right)=1$;

(b) if $\mathbb{M} \not \subset X_{1}$ (i.e., $\mathbb{M}_{2} \neq\{0\}$ ), then (by Lemma 5.2)

$$
\hat{\delta}\left(\mathbb{M}, X_{1}\right) \geq(1 / \gamma) \hat{\delta}\left(P_{2} \mathbb{M}, P_{2} \chi_{1}\right)=(1 / \gamma) \hat{\delta}\left(M_{2},\{0\}\right)=1 / \gamma,
$$

where $\gamma=\max \left(\left\|P_{1}\right\|,\left\|P_{2}\right\|\right)$.

Hence, $X_{1}$ and $X_{2}$ are isolated points of $\mathcal{I}$ and therefore $x_{1}, x_{2} \in \mathcal{I}^{\prime}$ (The orem 4.3).

Finally, observing that $g_{j}$ is $\hat{d}$-isometric to a closed subset of the metric space $(\mathscr{G}, \hat{d})(j=1,2)$, it is not hard to prove that $I_{=} \mathscr{I}_{1} \times \mathscr{I}_{2}$ as a topological spacè.

(v) Since $X_{1}, X_{2} \in \mathcal{I}$, it follows that $P_{1}, P_{2} \in \mathfrak{U}^{\prime}$ and, therefore (by Lemma 5.1), $X_{j}=P X_{j} \in \mathcal{I}^{\prime \prime}, j=1,2$; repeating the argument with $\mathscr{X}^{X}$ replaced by $\mathscr{X}^{\prime}$ and $\mathfrak{U}^{\prime}$ replaced by $\mathfrak{U}^{\prime \prime}=\left(\mathfrak{U}^{\prime}\right)^{\prime}$ we conclude that $P_{1}, P_{2} \in \mathfrak{U}^{\prime} \cap \mathfrak{U}^{\prime \prime}$. According to (i), this implies that $\mathfrak{U}^{\prime}$ and $\mathfrak{U}^{\prime \prime}$ (recall that $\left(\mathfrak{U}^{\prime \prime}\right)^{\prime}=\mathfrak{U}^{\prime}$ ) splits w.r.t. $\mathfrak{X}_{1}, \mathfrak{X}_{2}$.

The remaining statements are clear. Q.E.D.

If $X_{=} X_{1} \oplus X_{2}$ and $\mathfrak{X}=\mathscr{L}(X)$, then $\mathscr{I}=\{\{0\}, \mathscr{X}\}$ clearly splits w.r.t. $X_{1}, X_{2}$, but $X_{1}, X_{2} \notin g$ (unless one of these subspaces is trivial). We do not know whether the condition $X_{1}, X_{2} \in I$ permits one to "reverse" the statement (iii) of Theorem 5.3; this is possible under some additional hypothesis. 
Theorem 5.4. Let $X_{=} X_{1} \oplus X_{2}, X_{1}, X_{2} \in \mathcal{I}$ and assume that $\mathfrak{X}$ is relatively reflexive w.r.t. $\mathfrak{U}^{\prime} \cap \mathfrak{X}^{\prime \prime}$; then $\mathfrak{A}$ splits w.r.t. $\mathfrak{X}_{1}, \mathscr{X}_{2}$ if and only if $I$ splits w.r.t. $x_{1}, x_{2}$.

Proof. Assume that $X_{1}, X_{2} \in I$ and $I$ splits; then, by Theorem 5.3 (iv) and (v), $P_{j} \in \mathfrak{U}^{\prime} \cap \mathfrak{U}^{\prime \prime}, j=1,2$. From Theorem 5.3 (ii), we also see that $P_{j} \in \mathfrak{U} \hat{\text {; }}$ therefore $P_{j} \in \mathfrak{U}^{\wedge} \cap \mathfrak{U}^{\prime} \cap \mathfrak{U}^{\prime \prime}=\mathfrak{U} \cap \mathfrak{U}^{\prime}$. Now, the result follows from Theorem 5.3(i). Q.E.D.

Remarks. (a) If $\mathfrak{X}\left(=\mathfrak{U}_{1} \oplus \mathfrak{U}_{2}\right)$ splits, then $\mathfrak{U}^{\prime}=\mathfrak{U}_{1}^{\prime} \oplus \mathfrak{U}_{2}^{\prime}$, where $\mathfrak{U}_{j}^{\prime}$ is precisely the commutant of $\mathscr{U}_{j}, j=1,2$.

(b) In general, it is not true that the condition " $X_{1}, X_{2} \in I \cap g^{\prime}$ " implies " $g$ splits w.r.t. $X_{1}, X_{2}$." In fact, if $\mathscr{A}=\mathscr{U}_{T}$ is as in Example 2 and $K=K_{1} U$ $K_{2}$ is a disjoint union of $K$ into two measurable subsets of positive measure, then $X_{j}=L^{2}\left(K_{j}, \mu\right) \in \mathscr{I} \cap \mathcal{I}^{\prime}=\mathscr{I}_{T}^{\prime}$, but $\mathscr{I}$ cannot be decomposed (e.g., $H^{2} \cap$ $X_{j}=\{0\}$, for $\left.j=1,2\right)$.

(c) If $\mathscr{X}, \mathbb{B}, \mathfrak{S}$ are subalgebras of $\mathscr{L}(\mathscr{X})$ suth that $\mathscr{U} \subset \mathbb{B} \subset \mathbb{C} \subset \mathfrak{U}^{\prime \prime}$ and $\mathbb{B}$

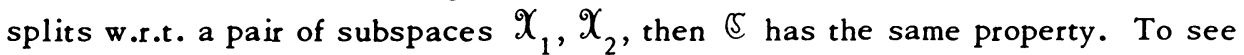
this, observe that, if $P_{1}$ is the projection of $X_{\text {onto }} X_{1}$ along $X_{2}$, then $P_{1}, P_{2}$ $\in \mathfrak{B} \cap \mathfrak{B}^{\prime}$; hence $P_{1}, P_{2} \in \mathfrak{C}$. Since $\mathfrak{A} \subset \mathfrak{U}^{\prime \prime}$ implies that $\mathfrak{U}^{\prime}=\mathfrak{B}^{\prime}=\mathbb{S}^{\prime}$, it follows that $P_{1}, P_{2} \in \mathbb{S} \cap \mathcal{S}^{\prime}$, from which the result follows.

Corollary 5.5. If $T \in \mathfrak{L}(\mathfrak{X}), \sigma(T)=\sigma_{1} \cup \sigma_{2}$ (disjoint union into two compact subsets) and $P_{1}, P_{2}$ are the projections associated with $\sigma_{1}, \sigma_{2}$, resp., then $X_{j}$ $=P{ }_{j} X \in \mathfrak{I}_{T}^{\prime}(j=1,2), \mathfrak{U}_{T}^{a}, \mathfrak{X}_{T}^{\prime \prime}, \mathfrak{X}_{T}^{\prime}$ split w.r.t. $\mathfrak{X}_{1}, X_{2}$ and the corresponding lattices $\mathcal{J}_{T}^{a}, \mathfrak{G}_{T}^{\prime \prime}, \mathcal{I}_{T}^{\prime}$ split w.r.t. this pair of subspaces.

In addition, if $P_{1}, P_{2} \in \mathfrak{U}_{T}$, then the analogous results are true for $\mathfrak{U}_{T}$ and $g_{T}$

Proof. $P_{1}, P_{2} \in \mathscr{U}_{T}^{a} \subset \mathfrak{U}_{T}^{\prime \prime} \subset \mathfrak{Q}_{T}^{\prime}$ (see [11, p. 419]). Q.E.D.

Remark. The disconnectedness of $\sigma(T)$ is not necessary for $\mathfrak{A}_{T}$ to split w.r.t. a pair of nontrivial subspaces. In fact, if $T$ is a normal operator in a Hilbert space $\mathcal{H}$, and $\sigma(T)=\sigma_{1} \cup \sigma_{2}$ is a disjoint union of $\sigma(T)$ into two Borel subsets such that the associated spectral projections $\left(P_{1}, P_{2}\right)$ are nontrivial, then $\mathcal{H}_{j}=P_{j} \mathcal{H}_{\in} \in \mathcal{I}_{T}^{\prime}$ and $\mathscr{U}_{T}$ splits w.r.t. $\mathcal{H}_{1}, \mathcal{H}_{2}$. However, for a suitable normal operator $T$ it is possible to get a partition of $\sigma(T)$ such that $\operatorname{cl}\left(\sigma_{1}\right) \cap$ $\operatorname{cl}\left(\sigma_{2}\right) \neq \varnothing$.

Given a compact subset $K$ of the plane, the polynomial hull $K^{\wedge}$ of $K$ is the complement of the unbounded component of the complement of $K$.

Corollary 5.6 (Crimmins and Rosenthal [5]). If $\sigma(T)=\sigma_{1} \cup \sigma_{2}$ and $\hat{\sigma}_{1} \cap$ $\hat{\sigma}_{2}=\varnothing$, then every invariant subspace $\mathbb{N}$ of $T$ bas a unique decomposition of 
the form $\mathbb{M}_{=} \mathbb{M}_{1} \vee \mathbb{M}_{2}$, where $\mathbb{M}_{j} \in \mathcal{I}_{T}$ and $\mathbb{M}_{j} \subset X_{j}=P_{j} X_{j}=1,2\left(P_{j}\right.$ is the projection associated to $\sigma_{j}$ ).

Proof. Let $V_{1}$ and $V_{2}$ be two disjoint compact neighborhoods of $\hat{\sigma}_{1}$ and $\hat{\sigma}_{2}$ such that $\hat{V}_{1} \cap \hat{V}_{2}=\varnothing$. By Runge's theorem, the analytic function (on interior $\left.\left(V_{1} \cup V_{2}\right)\right)$ which is equal to one on int $\left(V_{1}\right)$ and zero on int $\left(V_{2}\right)$ can be uniformly approximated by polynomials on $\hat{\sigma}_{1} \cup \hat{\sigma}_{2}$. Therefore $P_{1}, P_{2} \in \mathfrak{U}_{T} \subset$ $\mathscr{U}_{T}^{\prime}$, from which the result follows. Q.E.D.

Remark. The condition $\hat{\sigma}_{1} \cap \hat{\sigma}_{2}=\varnothing$ is not necessary for the idempotents associated with $\sigma_{1}$ and $\sigma_{2}$ to be in $\mathfrak{U}_{T}$. In fact, if, in Example 1, we take $q(z)$ $=z p(z)$, where $p(z)$ is a singular inner function whose natural boundary is the unit circle, then $\sigma(T)=\sigma_{1} \cup \sigma_{2}$, where $\sigma_{1}=\{0\}$ and $\sigma_{2}=\{z:|z|=1\}$, and therefore $\hat{\sigma}_{1} \cap \hat{\sigma}_{2} \neq \varnothing$. However, $\mathfrak{U}_{T}^{\prime}=\mathscr{U}_{T}$ contains the idempotents associated with $\sigma_{1}$ and $\sigma_{2}$.

6. Quotients and restrictions.

Theorem 6.1. Let $\pi \in \mathcal{I}_{T}, R=\left.T\right|_{\Re(\pi}$ and let $\pi$ be a subspace of $\Re$. Then the following bold:

(i) $\pi \in g_{R}$ if and only if $\pi \in g_{T}$. Moreover, $g_{R}$ can be identified with the closed subset

$$
\left\{\pi \in \mathscr{G}_{T}: \pi \subset \pi\right\} \subset \mathscr{G}_{T} .
$$

This identification is an isometry (with respect to the metrics $\hat{d}$ ).

(ii) If $\pi \in \mathcal{G}_{T}^{a}$ and $\pi \in \mathcal{G}_{R}^{a}$, then $\pi \in \mathcal{G}_{T}^{a}$.

(iii) If $\pi \in \mathcal{I}_{T}^{\prime}$ and $\pi \in \mathcal{I}_{R}^{\prime}$, then $\pi \in \mathcal{I}_{T}^{\prime}$.

Proof. The statement (i) is immediate.

(ii) If $\Re_{\in} g_{T}^{a}, \Re_{\in} g^{a}$, then (by Lemma 2.2) $\sigma(R) \subset \sigma(T)$ and $\sigma\left(\left.R\right|_{\Re}\right) \subset \sigma(R)$, and therefore $\sigma\left(\left.R\right|_{\Re}\right)=\sigma\left(\left.T\right|_{\pi}\right) \subset \sigma(T)$. Hence (by Lemma 2.2), $\pi \in \mathcal{G}_{T}^{a}$.

(iii) If $B \in \mathfrak{U}_{T}^{\prime}$ and $\mathbb{M} \in \mathscr{I}_{T}^{\prime}$, it is clear that $\left.B\right|_{\pi} \in \mathfrak{U}_{R}^{\prime}$ and therefore $\pi \in$ $\left.I_{B}\right|_{\pi}$, for all $\pi \in \mathcal{I}_{R}^{\prime}$; according to (i) this implies that $\pi \in \mathscr{I}_{B}$.

Since $\mathcal{I}_{T}^{\prime}=\bigcap\left\{\mathcal{G}_{B}: B \in \mathfrak{U}_{T}^{\prime}\right\}$, it follows that $\mathcal{I}_{R}^{\prime} \subset \mathcal{G}_{T}^{\prime}$. Q.E.D.

Theorem 6.2. Let $\mathbb{M} \in \mathcal{I}_{T}$ and let $\bar{T}$ be the operator in the quotient space $\bar{X}=X / M$ induced by T; i.e., if $\pi: \mathcal{X} \rightarrow \bar{X}$ is the canonical map of $\mathcal{X}$ onto its quotient space, then

$$
T(\pi x)=\pi(T x) .
$$

Let $\bar{\Re}$ be a subspace of $\bar{X}$ and let $\pi=\pi^{-1}(\bar{\gamma})$ be the inverse image (which is a subspace of $\mathcal{X}$ ) of $\bar{\pi}$ in $\mathcal{X}$. Then

(i) $\bar{r} \in \mathscr{I}_{\bar{T}}$ if and only if $\pi \in \mathfrak{I}_{T}$. 
Moreover, $\mathcal{I}_{\bar{T}}$ can be identified with the closed subset

$$
\left\{\pi \in \mathcal{I}_{T}: \Re \supset \mathbb{M}\right\} \mathfrak{I}_{T} .
$$

This identification is a bomeomorphism of topological spaces (with respect to the topologies induced by the metrics $\hat{d}$ ).

(ii) If $\pi \in \mathcal{I}_{T}^{a}$ and $\bar{r} \in \mathcal{I}_{T}^{a}$, then $\pi \in \mathcal{I}_{T}^{a}$.

(iii) If $\pi \in \mathcal{S}_{T}^{\prime}$ and $\bar{\Re} \in \mathcal{I}_{T}^{\prime}$, then $\Re \in \mathcal{S}_{T}^{\prime}$.

The first part of the proof of (i) follows immediately from (6.1). It is also clear that $\mathscr{I}_{T}$ can be identified with $\left\{\Re \in I_{T}: \Re \supset \Re\right\}$, as a partially ordered set. Finally, observe that if $\pi, \mathfrak{L} \in \mathscr{I}_{T}, \pi \cap \mathscr{L}^{\prime} \supset \mathbb{M}$, then

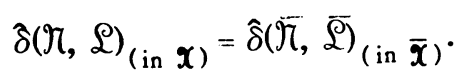

Using this equality and (4.1) the result follows. To complete the proof we shall need the following lemma:

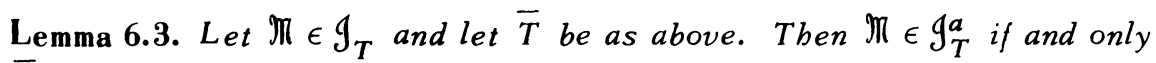
if $\sigma(\bar{T}) \subset \sigma(T)$.

Proof. Let $\mathbb{R} \in \mathscr{I}_{T} \backslash \mathcal{I}_{T}^{a}$; then (Lemma 2.2) there exist $\lambda \epsilon \rho(T)$ such that $\pi=(T-\lambda)^{-1} \pi \not \subset \pi$. Since $(T-\lambda) \pi \subset \pi$, it follows that $\pi \varsubsetneqq \pi$ and $(T-\lambda) \pi=$ M.

$\Re \in \mathscr{I}_{T}$ and therefore $\bar{\gamma}=\pi(\Re) \in \mathcal{I}_{\bar{T}}$ (Theorem 6.2(i)); moreover, $\pi[(T-\lambda) \Re]$ $=\pi \mathbb{M}=\{\overline{0}\}$. It is not hard to conclude that $\bar{\Re}=\operatorname{ker}(\bar{T}-\lambda) \neq\{\overline{0}\}$; hence $\lambda \in \sigma(\bar{T})$.

Now assume that $\mathbb{M} \in \mathscr{G}_{T}^{a}$; it is obvious that, if $(T-\lambda)$ maps $X$ onto $X$, then $(\bar{T}-\lambda)$ maps $\bar{X}$ onto $\bar{X}$. Moreover, if $\lambda \in \rho(T)$, then $(T-\lambda) \mathbb{M}=\mathbb{M}$ and this implies that $(\bar{T}-\lambda)$ is one-to-one. Hence $\sigma(\bar{T}) \subset \sigma(T)$. Q.E.D.

Now we can complete the proof of Theorem 6.2.

(ii) Let $\prod_{\epsilon} \in \mathcal{I}_{T}^{a}, \bar{\pi}=\mathcal{I}_{T} \frac{a}{T}$ and $\lambda \in \rho(T)$; by Lemma $6.3, \lambda \in \rho(\bar{T})$ and therefore $(\bar{T}-\lambda)^{-1} \bar{\gamma}=\bar{\gamma}$; hence $\pi=\pi^{-1}(\bar{\gamma}) \supset(T-\lambda)^{-1} \Re$; i.e., $\Re \subset(T-\lambda) \Re$.

On the other hand, (i) implies that $(T-\lambda) \Re \subset \Re$; hence $\Re=(T-\lambda) \Re=$ $(T-\lambda)^{-1} \Re$. Since this is true for all $\lambda \in \rho(T)$, we conclude that $\Re \in \mathcal{I}_{T}^{a}$.

(iii) Let $\pi \in \mathcal{I}_{T}^{\prime}$ and let $B \in \mathfrak{U}_{T}^{\prime}$. The operator $\bar{B}$ defined by $\bar{B}(\pi x)=\pi(B x)$ belongs to $\mathfrak{U} \frac{\prime}{T}$; therefore, if $\bar{r} \in \mathcal{I}^{\prime} \bar{T}=\bigcap\left\{\mathscr{G}_{L}: L \in \mathfrak{U} \frac{\prime}{T}\right\}$, then $\bar{\Re} \in \bigcap\left\{\mathcal{I}_{B}: B \in \mathfrak{U}_{T}^{\prime}\right\}$. Using this fact and (i), it follows that

$$
r \in \cap\left\{\mathscr{I}_{B}: B \in \mathfrak{U}_{T}^{\prime}\right\}=\mathscr{I}_{T}^{\prime} . \quad \text { Q.E.D. }
$$

Remark. It is natural to ask whether the same statements of Theorems 6.1 and 6.2 hold in the case of $g_{T}^{\prime \prime}$. We do not know the answer to that question, even in the case when $\mathbb{N}$ is assumed to be a hyperinvariant subspace. On the other hand, none of the statements of (ii) and (iii) of Theorems 6.1 and 6.2 can be "reversed"; in fact, we have 
Example 6. Let $U$ and $S$ be the bilateral and the unilateral shift (both defined as "multiplication by $\left.e^{i x \prime \prime}\right)$ acting on $L^{2}(\partial D)(D=\{z:|z|<1\})$ and $H^{2}$, respectively, and let $T=U \oplus S \in \mathfrak{L}\left(L^{2} \oplus H^{2}\right)$. Then $\sigma(T)$ is the closed unit disc $\bar{D}$ and therefore $\mathfrak{Q}_{T}^{a}=\mathfrak{U}_{T}$ (and $\mathcal{I}_{T}^{a}=\mathcal{I}_{T}$ ). We have

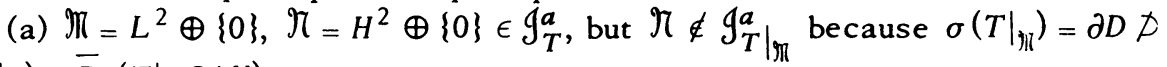
$\sigma\left(\left.T\right|_{\Re}\right)=\bar{D}\left(\left.T\right|_{\pi} \cong U\right)$

(b) $\mathbb{M}=\{0\} \oplus H^{2}, \pi=H^{2} \oplus H^{2} \in \mathcal{G}_{T}^{a}$, but $\bar{r} \notin \mathcal{G}_{T} \frac{a}{T}$ because $\sigma(\bar{T})=\partial D^{\prime} \not \supset$ $\sigma\left(\left.\bar{T}\right|_{\bar{r}}\right)\left(\bar{T} \cong U ;\left.\bar{T}\right|_{\bar{r}} \cong S\right)$.

Example 7. Let

$$
T=\left[\begin{array}{lll}
0 & 1 & 0 \\
0 & 0 & 0 \\
0 & 0 & 0
\end{array}\right]
$$

acting on $X=\bigvee\left\{x_{1}, x_{2}, x_{3}\right\}$; then $\mathfrak{U}_{T}^{\prime \prime}=\mathfrak{U}_{T}$ and, by Lemma 4.2,

$$
\begin{aligned}
& \Re=\bigvee\left\{x_{1}, x_{3}\right\}=\text { kernel } T \in \mathcal{I}_{T}^{\prime}, \\
& \mathbb{N}=\bigvee\left\{x_{1}\right\}=\text { range } T \in \mathcal{I}_{T}^{\prime} .
\end{aligned}
$$

\section{However}

(a) $R=\left.T\right|_{\Re}=0$ and therefore $\mathbb{M} \notin \mathscr{G}_{R}^{\prime}$.

(b) If $\bar{T}$ is defined as usual on $X / \dot{M}^{R}$, then $\bar{T}=0$ and $\bar{\gamma}=\pi(\Re) \notin \mathcal{Y}_{\bar{T}}^{\prime}$.

The restriction of $T$ to an invariant, but nonanalytically invariant subspace is very particular.

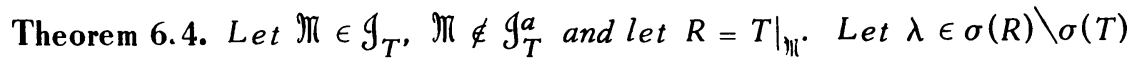
and define $\mathbb{M}_{\lambda, k}=(T-\lambda)^{k} \mathbb{M}$, for $k=0, \pm 1, \pm 2, \ldots$. Then

(i) $\lambda \in \sigma\left(\left.T\right|_{\prod_{\lambda, k}}\right) \backslash \sigma(T)$, for all integral $k$, and $\mathbb{M}_{\lambda, k} \varsubsetneqq \mathbb{M}_{\lambda, b}$ for all $k>b$.

(ii) If $\mathbb{M}_{\lambda}=\bigcap_{k=-\infty}^{+\infty} \mathbb{M}_{\lambda, k}$, then $\lambda \notin \sigma\left(\left.T\right|_{\pi_{\lambda}}\right)$.

Moreover, if $\mu$ belongs to the component of $\lambda$ in $\rho(T)$ and $\mathbb{M}_{\mu, k}=(T-\mu)^{k} M$ (k integral), $\mathbb{M}_{\mu}=\bigcap_{k=-\infty}^{+\infty} \mathbb{M}_{\mu, k}$, then $\mathbb{M}_{\lambda_{1}}=\mathbb{M}_{\mu}$.

(iii) Similarly, if $\Re_{\lambda}=\bigvee_{k=-\infty}^{+\infty} \pi_{\lambda, k}, \Re_{\mu}=\bigvee_{k=-\infty}^{+\infty} \pi_{\mu, k}$, then $\Re_{\lambda}=\Re_{\mu}$ and $\lambda \notin \sigma\left(\left.T\right|_{r_{\lambda}}\right)$.

(iv) $g_{R}^{\prime}$ contains a denumerable chain of subspaces. $X / M$.

Moreover, the same result is true for $\mathcal{G}_{\bar{T}}^{\prime}$, where $\bar{T}$ is defined by (6.1) on

Proof. (i) If $\mathbb{M}_{\lambda, 1}=\mathbb{M}_{\lambda, 0}(=\mathbb{M})$, then $\mathbb{M}_{=}(T-\lambda)^{-1} \mathbb{M}_{\text {and therefore } \lambda \epsilon}$ $\rho(R)$, contradicting our hypothesis; hence, $\mathbb{M}_{\lambda, 1} \neq \mathbb{M}_{\lambda, 0}$. Similarly, we can prove that $\mathbb{M}_{\lambda,-1} \neq \mathbb{M}_{\text {and }}$, by induction, we conclude that $\mathbb{M}_{\lambda, k} \varsubsetneqq \mathbb{M}_{\lambda, b}$ for all $k>b$. It is clear that $\lambda \in \sigma\left(\left.T\right|_{\mathbb{M}_{\lambda, k}}\right)$, for all $k$.

(ii) Observe that $(T-\lambda) \mathbb{M}_{\lambda}=\mathbb{M}_{\lambda}$ and therefore $\mathbb{M}_{\lambda}=(T-\lambda)^{-1} \mathbb{M}_{\lambda}$. Hence, $\mathbb{M}_{\lambda}$ is invariant under $(T-\lambda)$ and $(T-\lambda)^{-1}$, and $\lambda \notin \sigma\left(\left.T\right|_{\pi_{\lambda}}\right)$. It follows (see 
comments following Lemmas 2.1 and 2.2) that $\mathbb{M}_{\lambda}$ is invariant under $(T-\mu)^{-1}$ and $\mu \notin \sigma\left(\left.T\right|_{\pi_{\lambda}}\right)$; thus $(T-\mu)^{k} \mathbb{M}_{\lambda}=\mathbb{M}_{\lambda}$, for all integers $k$, and

$$
\mathbb{M}_{\lambda}=\bigcap_{k=-\infty}^{+\infty}(T-\mu)^{k} \mathbb{M}_{\lambda} \subset \bigcap_{k=-\infty}^{+\infty}(T-\mu)^{k} \mathbb{M}^{+}=\mathbb{M}_{\mu} .
$$

By symmetry, we get $\mathbb{M}_{\lambda}=\mathbb{M}_{\mu}$.

(iii) This can be proved by a formal repetition of the argument given in (ii).

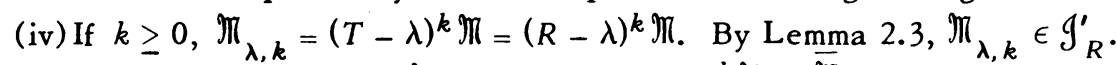

Similarly, if $k \geq 0$, then $\pi\left[M_{\lambda,-k}\right]=\pi\left[(T-\lambda)^{-k} M\right]=\bar{M}_{\lambda,-k}$ is the kernel of $(\bar{T}-\lambda)^{k}$; by Lemma $2.3, \bar{M}_{\lambda,-k} \in \bar{Y}_{\bar{T}}^{-k}$. It is clear that $\left\{\bar{M}_{\lambda,-k}\right\}_{k=0}^{\infty}$ is a chain of different hyperinvariant subspaces of $\bar{T}$. Q.E.D.

Let $\mathbb{N} \in \mathscr{G}_{T} \backslash g_{T}^{a}, R=\left.T\right|_{\pi}$ and let $\cong=\{p(z) / q(z): p, q$ are coprime monic polynomials with zeroes in $\sigma(R) \backslash \sigma(T)\}$. $\supseteq$ is a partially ordered set under the relation $p_{1} / q_{1}<p_{2} / q_{2}$ if and only if $\left(p_{1} q_{2}\right) /\left(p_{2} q_{1}\right)$ is a polynomial.

Theorem 6.5. The mapping $\Phi: \cong \rightarrow g_{T}$ defined by

$$
\Phi(p / q)=p(T)[q(T)]^{-1 M}
$$

is an order isomorphism from $\cong$ into $\mathscr{G}_{T} \backslash g_{T}^{a}$.

Proof. (1) Let $\lambda, \mu \in \sigma(R) \backslash \sigma(T), \lambda \neq \mu$, and let

$$
\mathbb{M}_{\lambda}=(T-\lambda) M, \quad \mathbb{M}_{\mu}=(T-\mu) M,
$$

$\mathbb{M}_{\lambda}=\mathbb{M}_{\mu}$, if and only if $\mathbb{M}_{=}(T-\lambda)^{-1}(T-\mu) M_{=}=\left[I+(\lambda-\mu)(T-\lambda)^{-1}\right] \mathbb{M}$; therefore, $\mathbb{M}_{\lambda}=\mathbb{M}_{\mu}$ implies that $(T-\lambda)^{-1} \mathbb{M} \subset \mathbb{M}$, a contradiction. We conclude that $\pi_{\lambda} \neq \pi_{\mu}$.

(2) Clearly, $(T-\lambda)(T-\mu) \mathbb{M}_{\lambda} \subset \mathbb{M}_{\lambda} \cap \mathbb{M}_{\mu}$.

On the other hand, if $\lambda \neq \mu$ and $x \in \mathbb{M}_{\lambda} \cap \mathbb{M}_{\mu}$, then $x=(T-\lambda) y=(T-\mu) z$, where $y, z \in \mathbb{M}$. Hence

$$
y=(T-\lambda)^{-1} x=(T-\lambda)^{-1}(T-\mu) z=\left[I+(\mu-\lambda)(T-\lambda)^{-1} z\right] \in \mathbb{M}
$$

and therefore $(T-\lambda)^{-1} z \in \mathbb{M}$; i.e., $z \in \mathbb{M}_{\lambda}$. Thus, we obtain

$$
x=(T-\mu) z=(T-\mu)(T-\lambda) z^{\prime}, \quad \text { for some } z^{\prime} \in \mathbb{M}
$$

i.e., $x \in(T-\lambda)(T-\mu) M$. We conclude that, if $\lambda \neq \mu$, then

$$
(T-\lambda)(T-\mu) \mathbb{M}=\mathbb{M}_{\lambda} \cap \mathbb{M}_{\mu} \text {. }
$$

(3) Combining these two results and Theorem 6.4(i), it is possible to show that $\Phi$, restricted to the polynomials contained in $\Omega$, is an injective order-preserving map. Then, the final result follows immediately. Q.E.D. 
We close this last section of the paper by studying the case of a subnormal ope rator.

Definition 6.1. An operator $R$ acting on a Hilbert space $\mathcal{K}$ is called subnormal if there exist a Hilbert space $\mathcal{H} \supset \mathcal{K}$ and a normal operator $T \in \mathscr{L}(\mathcal{H})$ such that $K \in \mathcal{G}_{T}$ and $R=\left.T\right|_{K}$.

Given a subnormal operator $R$ there always exists a (unique, up to unitary transformations) minimal normal extension $T$ in the sense that

$$
\mathcal{H}=\bigvee \mathfrak{X}_{T}^{\prime \prime}(\mathcal{K})=\bigvee_{k=0}^{\infty}\left\{T^{k}(\mathcal{K}), T^{* k}(\mathcal{K})\right\}
$$

Moreover, $\sigma(T) \subset \sigma(R)$ (see [8]).

Example 8. Let $\mu$ be a (Borel) positive measure on $\mathrm{C}$ with compact support $K$, and let

$R^{2}(K, \mu)=L^{2}(K, \mu)$ - closure of the set of all rational functions with poles outside $K$;

$H^{2}(K, \mu)=L^{2}(K, \mu)$ - closure of the polynomials.

Let $T=$ "multiplication by $z$ " on $L^{2}(K, \mu)$ and let $R=\left.T\right|_{R^{2}}$ and $H=\left.T\right|_{H^{2}}$. Then $R$ and $H$ are subnormal operators with minimal normal extension $T$.

In [2] (see also [19]), J. Bram proved that any subnormal operator $L$ with a cyclic vector (i.e., there exists $x \in \mathcal{K}$ such that $\mathcal{K}=\bigvee_{k=0}^{\infty} L^{k} x$ ) is unitarily equivalent to an operator of the form $H$ in the above example.

The existence of analytically invariant subspaces for a class of operators which include the case of the operator $R$ of the example, as a particular case, was studied by J. Brennan and J. Wermer [3], [4]. Here we present a "Bram's type theorem" for analytically invariant subspaces of subnormal operators.

Theorem 6.6. Let $\mathcal{K}$ be a Hilbert space of dimension greater than one. The following two statements are equivalent:

(1) $g_{S}^{a}$ is nontrivial for any subnormal operator $S$.

(2) $g_{R}^{a}(R$ given by Example 8$)$ is nontrivial.

Proof. (1) $\Rightarrow(2)$ is a trivial implication.

(2) $\Rightarrow$ (1) Let $S$ be a subnormal operator and let $T$ be its minimal normal extension. Then $S=\left.T\right|_{\mathcal{K}}, T \in \mathscr{L}(\mathcal{H})$ and $\sigma(T) \subset \sigma(S)$.

(a) If $\sigma(T) \neq \sigma(S)$, then (by Theorem 6.4 (iv)) $\mathcal{G}_{S}^{\prime}$ contains infinitely many elements.

So, we can assume that $\sigma(T)=\sigma(S)$.

(b) Let $x$ be a nonzero vector of $\mathcal{K}$ and let $\mathcal{K}=\mathrm{V}\left\{B x: B \in \mathfrak{U}_{T}^{a}\right\} \in \mathcal{G}_{T}^{a}$.

Claim. $\pi \in \mathcal{I}_{S}^{a}$.

In fact, if $\Re_{\in} g_{T}^{a}$ then (by Lemma 2.2) $\Re_{\in} \mathscr{G}_{S}$ and $\sigma\left(\left.T\right|_{r}\right) \subset \sigma(T)=\sigma(S)$; 
thus $\pi \in \mathcal{I}_{S}, \sigma\left(\left.S\right|_{\Re}\right) \subset \sigma(S)$, and (by Lemma 2.2) $\pi \in \mathcal{I}_{S}^{a}$. If $\Re \neq \mathcal{K}$, we are done. Otherwise

$$
\mathcal{K}=\Re \subset \Re^{\prime}=\bigvee_{k, b=0}^{\infty}\left\{T^{* k} T^{b} x\right\}=\bigvee\left\{B x: B \in \mathscr{U}_{T}^{\prime \prime}\right\} \in \mathcal{I}_{T}^{\prime \prime} \subset \mathcal{I}_{T}^{a} .
$$

Since $T$ is the minimal normal extension, $\mathcal{H}^{\prime}$ must be equal to $\mathcal{H}$. In this case (see [17]), $T$ acting in $\mathcal{H}$ is an operator of the type described in Examples $2,3,4$ ( $T$ is unitarily equivalent to "multiplication by $z$ " in a suitable $L^{2}(K, \mu)$ space; $K=\sigma(T)=\sup (\mu)), S=\left.T\right|_{\mathrm{K}}$ and

$$
\begin{gathered}
K=\bigvee\left\{B x: B \in \mathfrak{U}_{T}^{a}\right\}=L^{2}(K, \mu) \text { - closure of the rational functions with poles } \\
\text { outside } K ;
\end{gathered}
$$

i.e., $K=R^{2}(K, \mu)$. Q.E.D.

In the same lines as [19, Theorem 3 and Lemma 3.3] we can prove the following:

Theorem 6.7. If $R$ and $R^{2}(K, \mu)$ are defined as in Example 8, then

$$
\mathfrak{U}_{R}^{\prime}=\mathfrak{U}_{R}^{a}=\left\{M_{f}: f \in R^{2}(K, \mu) \cap L^{\infty}(K, \mu)\right\} .
$$

Moreover, if $A=M_{f} \in \mathfrak{U}_{R^{\prime}}^{\prime}$ then $\|A\|=\|f\|_{\infty}$.

In particular, this means that all the invariant subspaces of [4] are actually hyperinvariant.

Note. After this paper was written C. Pearcy and N. Salinas proved the following theorem, which generalizes a result of W. B. Arveson and J. Feldman.

Theorem. Let $T$ be a quasitriangular operator on a Hilbert space, and sup. pose that there exist a sequence of rational functions $\left\{r_{k}\right\}$ and a nonzero com. pact operator $K$ such that $\left\|r_{k}(T)-K\right\| \rightarrow 0$. Then $T$ bas a nontrivial analytically invariant subspace.

Moreover, this theorem is not vacuous in the sense that there exists a quasitriangular operator $T$ with the following properties:

(1) the spectrum of $T$ is connected;

(2) there exists no nonzero compact operator in the uniformly closed algebra generated by the polynomials in $T$; and

(3) there exists a quasinilpotent compact operator in the uniformly closed algebra generated by the rational functions in $T$.

Added in proof. Regarding to Lemma 2.3 and comments following it, R. Gellar and D. A. Herrero (Hyperinvariant subspaces of bilateral weighted shifts (preprint)) have proved that, for two large families of bilateral weighted shifts in Banach spaces, the kernel of every nonzero operator commuting with the shift is $\{0\}$ and the range is dense. 


\section{REFERENCES}

1. G. Birkhoff, Lattice theory, 2nd rev. ed., Amer. Math. Soc. Colloq. Publ., vol. 25, Amer. Math. Soc., Providence, R.I., 1948. MR 10, 673.

2. J. Bram, Subnormal operators, Duke Math. J. 22 (1955), 75-94. MR 16, 835.

3. J. E. Brennan, Invariant subspaces and rational approximation, J. Functional Analysis 7 (1971), 285 -310.

4. - Point evaluations and invariant subspaces, Indiana Univ. Math. J. 20 (1971), 879-881.

5. T. Crimmins and P. Rosenthal, On the decomposition of invariant subspaces, Bull. Amer. Math. Soc. 73 (1967), 97-99. MR 34 \# 3314.

6. R. G. Douglas and C. Pearcy, On topology for invariant subspaces, J. Functional Analysis 2 (1968), 323-341. MR 38 \#1547.

7. P. Halmos, Ten problems in Hilbert spaces, Bull. Amer. Math. Soc. 76 (1970), 887933. MR $42 \# 5066$.

8. - Normal dilations and extensions of operators, Summa Brasil. Math. 2 (1950), 125-134. MR 13, 359.

9. H. Helson, Lectures on invariant subspaces, Academic Press, New York, 1964. MR $30 \# 1409$.

10. T. Kato, Perturbation theory for linear operators, Die Grundlehren der math.

Wissenschaften, Band 132, Springer-Verlag, New York, 1966. MR 34 \#3324.

11. F. Riesz and B. Sz॰Nagy, Leçons d'analyse fonctionnelle, Akad. Kiadó, Budapest, 1953; English transl., Ungar, New York, 1955. MR 15, 132; MR 17, 175.

12. W. Rudin, Real and complex analysis, McGraw-Hill, New York, 1966. MR 35 \#1420.

13. D. Sarason, Generalized interpolation in $H^{\infty}$, Trans. Amer. Math. Soc. 127 (1967), 179-203. MR 34 \#8193.

14. - The $H^{p}$ spaces of an annulus, Mem. Amer. Math. Soc. No. 56 (1965). MR 32 \#6256.

15. - Invariant subspaces and unstarred operator algebras, Pacific J. Math. 17 (1966), 511-517. MR $33 \# 590$.

16. J. T. Schwartz, $\mathbb{W}^{*}$-algebras, Gordon and Breach, New York, 1967. MR 38 \#547.

17. I. E. Segal, Decompositions of operator algebras. I, II, Mem. Amer. Math. Soc. No. 9 (1951). MR 13, 534; 472.

18. B. Sz.-Nagy and C. Foias, Analyse harmonique des opérateurs de l'espace de Hilbert, Masson, Paris; Akad. Kiadó, Budapest, 1967. MR 37 \#778.

19. T. Yoshino, Subnormal operators with a cyclic vector, Tôhoku Math. J. (2) 21 (1969) 47-55. MR $39 \# 7450$.

DEPARTMENT OF MATHEMATICS, STATE UNIVERSITY OF NEW YORK AT ALBANY, ALBANY NEW YORK 12222

DEPARTMENT OF MATHEMATICS, UNIVERSITY OF MICHIGAN, ANN ARBOR, MICHIGAN 48104

Current address (D. A. Herrero): Universidade Estadual de Campinas, I.M.E.C.C., Caixã Postal 1170, 13100 Campinas, São Paulo, Brasil

Current address (Norberto Salinas): Department of Mathematics, University of Kansas, Lawrence, Kansas 66044 